\title{
Point source generation of chiral fields: Measures of near- and far-field optical helicity
}

\author{
Jamie M. Leeder, Henryk T. Haniewicz, David L. Andrews* \\ School of Chemistry, University of East Anglia, Norwich, NR4 7TJ, United Kingdom \\ *Corresponding author: david.andrews@physics.org
}

Received XX Month XXXX; revised XX Month, XXXX; accepted XX Month XXXX; posted XX Month XXXX (Doc. ID XXXXX); published XX Month XXXX

To consider the relationship between different measures of chirality in an optical field, the simplest case is considered: direct spontaneous emission of circularly polarized light by a point source. In the electromagnetic fields radiated from a suitably chiral source, such as a low-symmetry chiral molecule undergoing radiative decay, optical helicity is exhibited in the extent of a difference in left- and right-handed circular polarization components. There are several practical measures for quantifying the emergence of ensuing optical helicity, exhibiting different forms of dependence on the properties of the emitter and the positioning of a detector. By casting each measure in terms of an irreducible helicity density, connections and distinctions can be drawn between results expressible in either classical or quantum form. (C) 2015 Optical Society of America

OCIS codes: (160.1585) Chiral media; (260.3800) Luminescence; (260.5430) Polarization; (270.5580) Quantum electrodynamics

http://dx.doi.org/10.1364/AO.99.099999

\section{INTRODUCTION}

Building on foundational work by Lipkin, [1] a range of issues concerning the helicity of light - in particular its measures, conservation and connection to optical angular momentum - have attracted an increasing amount of interest in recent years. [2-8] It has, for example, been shown that an optical chirality density and its associated flux can both be defined in terms of helicity and spin. [9,10] Moreover it emerges that these each of these quantities, expressed in photonic terms, has a direct dependence on the relative occupancy of optical modes with opposing helicity - a feature that is most readily apparent when left- and right-handed modes are used as the basis. [11,12] To further address in detail the specific relationships between various practical measures of optical chirality, it is appropriate to consider the very simplest system: the optical field associated with spontaneous, circularly polarized emission from an isolated point source. Focusing on quantitative analytical measures of optical helicity, the development of electrodynamic theory leads to expressions for three distinct observables, each of which has a connection with optical helicity. The results reveal how the evolution of these measures, as the light propagates outwards from the source, change from near-field to farfield form.

\section{DESCRIPTION OF THE SYSTEM}

Spontaneous photon emission by a single chiral molecule is the simplest optical process in which material chirality is conferred into an optical field - and to begin, it may be helpful to reflect on the physical mechanism involved. It may be assumed that the molecule, initially in an electronically excited state, has the same nuclear geometry as the ground states: the symmetry properties of those two states will be identical. There is no sense in which the photon released in its process of electronic decay 'conveys away' any molecular chirality, not least because the ground state molecule fully retains the chiral character of its initial, excited state. There is no meaningful measure of net helicity for a system comprising both matter and light. [13] However, in the initial state of the emission process, in which the radiation field is empty, the symmetry of the system is determined by the excited molecule generally a subgroup of the rotation group $\mathrm{SO}(3)$.

The expectation of differential circularity in the emission from a chiral molecule is perhaps most readily appreciated by considering the timeinverse process: temporal (and spatial) symmetry considerations afford fundamental insights into magnetoelectric chiral interactions. [22] It is well known that chiral molecules exhibit circular dichroism (CD): [1416] a range of important applications in absorption spectroscopy are based on this sensitivity to the handedness of input light. It is clear on symmetry grounds that there is a qualitative difference between the interactions of, say a 'left-handed' molecule with a left-handed photon, as opposed to a right-handed one this is manifest in a quantifiable CD a corresponding difference in absorption rate. From time-reversal arguments it therefore follows that chiral discrimination in spontaneous emission has a similar physical basis - indeed, the technique of circular polarized luminescence has developed from this exact principle. [16-19] Equally, symmetry arguments based on spatial inversion show that, just as molecules of one particular enantiomeric form can display a difference in rates for emitting left-, compared to right-, circular light, the same difference can be expected between the rates of emitted light of one specific optical circularity, from opposite enantiomers. The principle is illustrated in schematic form in Fig. 1. 


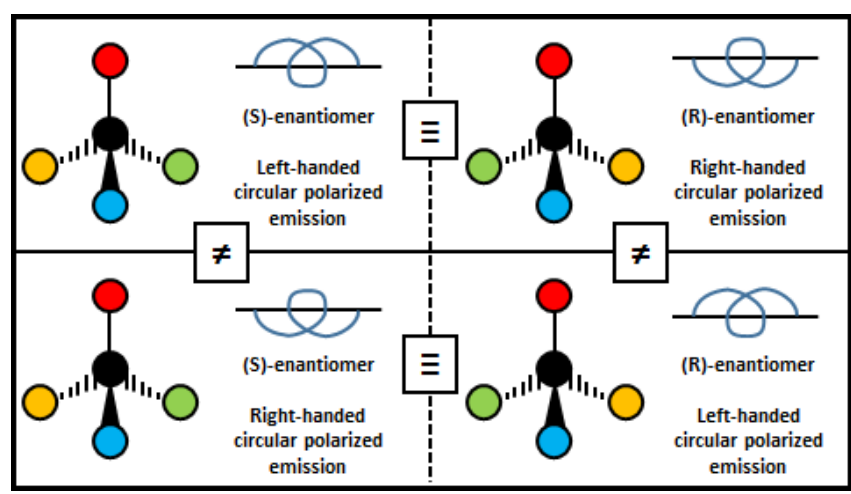

Fig. 1. (Color online) Correspondence and non-correspondence in the forms of interaction between enantiomers and circularly polarized photons of each handedness

\section{MEASURES OF OPTICAL HELICITY}

The fundamental measure of optical helicity in classical electrodynamics is most simply defined by the following formula:

$$
\kappa=\int \mathbf{A}(\mathbf{r}) \cdot \mathbf{B}(\mathbf{r}) d^{3} \mathbf{r},
$$

where $\mathbf{A}$ and $\mathbf{B}$ are the electromagnetic vector potential and magnetic induction fields, respectively. In the corresponding quantum field formulation, in which both of these fields are elevated to operator status and cast as Fourier series, it is not immediately apparent that the result is Hermitian, as should befit a quantum observable. However, on performing a mode expansion the helicity emerges in an obviously Hermitian form simply cast in terms of the number operators for left and right circular polarizations, $\hat{N}_{L}$ and $\hat{N}_{R}$ respectively: [12]

$$
\hat{\kappa}=\frac{\hbar}{\varepsilon_{0} c} \sum_{\mathbf{k}}\left(\hat{N}_{L}(\mathbf{k})-\hat{N}_{R}(\mathbf{k})\right),
$$

in which $\varepsilon_{0}$ is the vacuum permittivity, $\hbar$ is the reduced Planck constant and $\mathbf{k}$ represents a wave vector. For radiation propagating in a region sufficiently far away from a point source, a plane wave representation for each specific wave-vector is appropriate, and the corresponding intensity of light is suitably expressible in terms of a mean irradiance $I$. Duly re-expressing equation (1) in terms of photons of left- and righthanded polarizations, measurement of the optical helicity for a specific wave vector is therefore given by:

$$
\kappa=\frac{V}{\varepsilon_{0} c^{3} k}\left(I_{L}-I_{R}\right),
$$

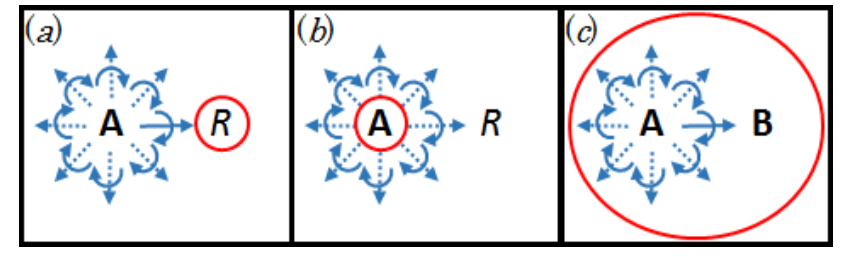

Fig. 2. (Color online) Three methods used to gauge, at a position $R$, the emission from a chiral molecule $A$ at the origin, curly arrows indicating the capacity of the source to radiate circularly polarized photons. Red lines figuratively indicate the focus of measurement. (a) Determination of a Stokes parameter at $R$; $(b)$ Identification of a differential in the rate of spontaneous emission by A; $(c)$ Measurement of a differential in the source emission through photon absorption by a physical detector $B$ at position $R$. where $V$ represents the volume around the point of detection. The main intention of this article is to now consider and duly assess different mathematical means by which objective, quantifiable measurements of radiation helicity can be made. Three methods are considered; the first involves one of the Stokes parameters, directly addressing optical circularity in terms of the polarization of the emergent field; the second quantifies the circular differential rate of spontaneous emission, as might be measured by an independent detector; the third treats the emitter and detector as optically coupled components. Each method is illustrated within Fig. 2.

\section{A. Helicity Measurement through Stokes Polarimetry}

The polarization of a single quantized mode is typically quantified in terms of four Stokes parameters $S_{n}$ - each one directly related to wave intensities measured in different orthogonal bases. With respect to the chirality focus of this investigation, the most significant of these operators is $S_{3}$, whose classical expression is commonly cast as; [20,21]

$$
\begin{aligned}
S_{3} & \equiv I_{R}-I_{L} \\
& =2 \operatorname{Im}\left[\bar{E}_{x} E_{y}\right] \equiv \operatorname{Im}\left[\bar{E}_{x} E_{y}-\bar{E}_{y} E_{x}\right],
\end{aligned}
$$

where each $\mathbf{E}$ is a complex vector associated with the electric field (but cast in different units). However, the corresponding expression for the third Stokes operator, in quantum form, is conventionally cast in a similar guise as follows; [22]

$$
\hat{S}_{3}=i\left(a_{y}^{\dagger} a_{x}-a_{x}^{\dagger} a_{y}\right) \text {. }
$$

in which $a$ and $a^{t}$ represent the familiar photon annihilation and creation operators respectively. To more clearly establish a connection to circular polarizations, use can be made of the following identities;

$$
a_{x}=\frac{1}{\sqrt{2}}\left(a_{L}+a_{R}\right) ; \quad a_{y}=\frac{i}{\sqrt{2}}\left(a_{L}-a_{R}\right) .
$$

By substitution, it is again possible to re-express equation (5) in terms of the number operators for left- and right- handed photon populations where $a_{L}^{\dagger} a_{L}-a_{R}^{\dagger} a_{R}=\hat{N}_{L}-\hat{N}_{R}$, leading to the sought connection:

$$
\hat{S}_{3}=\frac{\hat{\kappa} \varepsilon_{0} c}{\hbar} \text {. }
$$

For the corresponding relation between measurements, we can use the standard relation $I=N \hbar c^{2} k / V$ to connect irradiance with the expectation number of photons in volume $V$, so producing the result:

$$
\kappa=\frac{k}{\varepsilon_{0} c}\left(N_{L}-N_{R}\right) .
$$

In passing we note that the expression for $S_{3}$ based on the quantum formula therefore delivers a result of opposite sign to the classical expression (4). Nonetheless, it is clear from this that the third Stokes parameter does correctly register optical helicity. As a result of the analysis previously performed by Coles and Andrews, it therefore transpires that this correlation holds true even in the case of optical vortex radiation ('twisted beams'). However, the assumption of remoteness from the source (insignificantangle of divergence) suggests that the connection may hold true only in the far-zone (FZ), $\lambda<<R$ where $\lambda$ is the optical wavelength and $R$ the distance from the source. We now explore this issue in detail.

The classical, text-book expression (4) casts the result for $S_{3}$ in a form suited to plane wave detection, and both $E_{x}$ and $E_{y}$ can accordingly be determined from classical expressions. [23] In a general emissive field, the fully retarded electric field from an E1 (electric dipole) radiative transition is given as: 
$\mathbf{E}^{(E 1)}=\frac{\exp (i k R)}{R^{3}}\left\{k^{2} R^{2}(\hat{\mathbf{R}} \times \boldsymbol{\mu}) \times \hat{\mathbf{R}}+[3 \hat{\mathbf{R}}(\hat{\mathbf{R}} \cdot \boldsymbol{\mu})-\boldsymbol{\mu}](1-i k R)\right\}$,

with $\hat{\mathbf{R}}$ and $\boldsymbol{\mu}$ the unit displacement vector and electric dipole moment for the source transition, respectively. This formula accommodates both near- and far-field behavior. The corresponding electric field from an M1 (magnetic dipole) transition also follows as:

$$
\mathbf{E}^{(M 1)}=-\frac{\exp (i k R)}{R^{3}}(\hat{\mathbf{R}} \times \mathbf{m})\left(k^{2} R^{2}+i k R\right),
$$

introducing the magnetic transition dipole moment $\mathbf{m}$. For an electronically excited transmitter whose chirality permits radiative decay transition simultaneously allowed by both E1 and M1 selection rules, the total electric field is subsequently the sum of equations (9) and (10). Expressing all vectors in the resulting expression in Cartesian componentform, the $x$ component of the electric field is thus expressible as;

$$
E_{x}=\frac{\exp (i k R)}{4 \pi \varepsilon_{0} R^{3}}\left[-\mu_{x}\left(1-i k R-k^{2} R^{2}\right)+c^{-1} m_{y}\left(i k R+k^{2} R^{2}\right)\right] .
$$

This result is determined under the condition that 'measurement' of the field is made along the $z$-axis of the emission, therefore the displacement vectors projected along the $x$ and $y$ axes are both zero. Whilst the $y$ component of the electric field can also be extracted from the sum of equations (9) and (10), an alternative method involves rotating the Cartesian indices in equation (11) about the $z$-axis. By either prescription, $E_{y}$ is proven to be identical to equation (11) upon substitution of $\mu_{x}$ with $\mu_{y}$, and $m_{y}$ with $-m_{x}$. The $S_{3}$ parameter of an emerging electric field then follows by substitution of $E_{x}$ and $E_{y}$ into equation (4). In the far-zone regime, the parameter involves terms with an inverse square dependence on distance, whose measurement satisfies the required invariance to $z$-axis rotation:

$$
S_{3}^{(F Z)}=\frac{i k^{4}}{\left(4 \pi \varepsilon_{0} R\right)^{2} c}\left(\mu_{x} m_{x}+\mu_{y} m_{y}\right) .
$$

In the derivation of equation (12), it is assumed that the electric dipole moments are real, whereas the magnetic moments are entirely imaginary. For this to hold, the condition is that the spatial wavefunctions are real - which is always satisfied by non-degenerate states, or can be satisfied by suitably chosen combinations in the case of degenerate states. The physically appealing form of the $S_{3}$ parameter is thus consistent with a scalar product of $\boldsymbol{\mu}$ and $\mathbf{m}$, excluding their 'longitudinal' $z$-components. In the near-zone (NZ) limit, $R \ll \lambda$, it emerges that $S_{3}$ instead varies with $R^{-5}$,

$$
S_{3}^{(N Z)}=\frac{k}{\left(4 \pi \varepsilon_{0}\right)^{2} R^{5} c}\left(\mu_{x} m_{x}-\mu_{y} m_{y}\right) .
$$

Interestingly, the optical response determined by equation (13) exhibits a change in sign upon rotation about the $z$-axis, incompatible with the arbitrary designations of the $x$ and $y$ directions for the circularly polarized emitted field. Although the same issue might have been thought to apply to the right-hand side of the defining equation (4), the difference in (13) is that here the $x$ and $y$ directions are no longer arbitrary; in an emitter that is necessarily anisotropic they have specific significance as reference coordinates for components of the emission dipoles. More significantly, the result (13) is clearly imaginary, in light of the imaginary character of the magnetic transition moment. The result therefore validates the earlier suggestion that in the near-zone (in the vicinity of the source), the third Stokes parameter does not afford a valid representation of optical helicity.
Partly to corroborate the result, an alternative method of determining $S_{3}$, based on the formalism of QED, is now introduced. Here, the effective electric field generated by the source emission is determined in terms of components from the corresponding electric and magnetic decay terms, by deriving the coupling fields associated with photons of arbitrary propagation and polarization vector. Indeed, this requires adding the quantum amplitudes for the release of circularly polarized photons through source decay. Here, expressing the result with implied summation over repeated Cartesian indices, we have

$$
E_{j}=-\mu_{i} V_{i j}-c^{-1} m_{i} U_{i j},
$$

where $V_{i j}$ and $U_{i j}$ represent the second-rank E1-E1 and E1-M1 coupling tensors respectively; [24]

$$
\begin{aligned}
V_{i j} & =\frac{\exp (i k R)}{4 \pi \varepsilon_{0} R^{3}}\left[(1-i k R)\left(\delta_{i j}-3 \hat{R}_{i} \hat{R}_{j}\right)-k^{2} R^{2}\left(\delta_{i j}-\hat{R}_{i} \hat{R}_{j}\right)\right] \\
U_{i j} & =\frac{\exp (i k R)}{4 \pi \varepsilon_{0}} \varepsilon_{i j k} \frac{\hat{R}_{k}}{R^{3}}\left(-i k R-k^{2} R^{2}\right) .
\end{aligned}
$$

Upon substitution of the tensors in equation (15) into equation (14), it is possible to determine the $x$ and $y$ components of the emerging field, as before. For the given system, only diagonal components of the $V$ tensor yield non-zero electric contributions to the field, whilst for the $U$ tensor, non-zero components arise only for $i \neq j$, and neither index can represent the $z$-component, given that $R_{k}=R_{\mathrm{z}}$. Utilizing equation (4) now produces an $S_{3}$ parameter whose far-zone limit proves identical in form to equation (12). The non-physical nature of the near-zone result, noted earlier, therefore emerges as a reflection upon the short-range validity of the Stokes parameter, rather than any issue between a classical or quantum field formulation.

\section{B. Helicity Measured in Spontaneous Emission}

Having evaluated emission chirality as determined by the third Stokes parameter, an alternative, quantitative means to assess chiral discrimination, focusing on the spontaneous emission from the emitter, is considered. [25] Specifically, the differential rate of emission of photons possessing either left- or right-circular polarization is now to be directly investigated. It is appropriate to begin with the following expression for $I(\Omega)$, the radiant intensity per unit solid angle $\Omega$,

$$
I(\Omega) d \Omega=\Gamma \times \hbar c k,
$$

in which the emission is cast in terms of a product of the emission rate $\Gamma$, and the photon energy $\hbar c k$. The former, secured through the 'Fermi Golden Rule' is linked to the system matrix element $M_{F}$, the latter characterizing the quantum amplitude for progression from the initial system state $|I\rangle$ to the final state $|F\rangle$,

$$
\Gamma=\frac{2 \pi \rho_{F}}{\hbar}\left|M_{F I}\right|^{2} .
$$

In equation (17), $\rho_{F}$ represents the density of final states for the radiation field, which for the given system is written in the following form,

$$
\rho_{F}=\frac{k^{2} V d \Omega}{8 \pi^{2} \hbar c} .
$$

As before, emission is assumed to occur through decay transitions that are both $\mathrm{E} 1$ and M1 allowed, resulting in the following matrix element,

$$
M_{F I}^{(A)}=\left(\frac{\hbar c k}{2 \varepsilon_{0} V}\right)^{\frac{1}{2}}\left(i \bar{e}_{i} \mu_{i}+i c^{-1} \bar{b}_{i} m_{i}\right),
$$


where $e_{i}$ and $b_{i}$ represent components of the electric and magnetic polarization vectors respectively. The superscript notation on $M_{F I}^{(A)}$ identifies emitter $A$ as the focus of measurement. The rate of emission as determined by equation (17) invokes the square modulus of the emission matrix element,

$$
\begin{aligned}
\left|M_{F I}^{(A)}\right|^{2} & =\left(\frac{\hbar c k}{2 \varepsilon_{0} V}\right)\left[\bar{e}_{i} e_{j} \mu_{i} \bar{\mu}_{j}+c^{-2} \bar{b}_{i} b_{j} m_{i} \bar{m}_{j}\right. \\
& \left.+c^{-1}\left(\bar{e}_{i} b_{j} \mu_{i} \bar{m}_{j}+e_{i} \bar{b}_{j} \bar{\mu}_{i} m_{j}\right)\right] .
\end{aligned}
$$

This equation, of general applicability, relates to the rate of emission of a single emitter, at any given instant in time. To secure a time-averaged result for a system undergoing stochastic rotations, or equally an ensemble average for a system comprising randomly oriented emitters, a rotational average is implemented by an integration-free method utilizing isotropic tensors. [26,27] The average of equation (20) thus entails a Kronecker delta (the second-rank isotropic tensor) which duly acts to contract the dummy indices $i$ and $j$. The terms in the second line of equation (20) then vanish for linearly polarized light; however, for circularly polarized emission the result of effecting tensor contraction between the electric and magnetic polarization vectors is non-zero, and depends on the handedness of the emission. The reason is that the radiation and molecular parts of each term in the round brackets - for example $\bar{e}_{i} b_{j}$ and $\mu_{i} \bar{m}_{j}$ - are both polar second-rank tensors, whose sign changes on spatial inversion. Utilizing the standard spherical basis vectors for left- and right polarized light, the rotationally averaged matrix element for spontaneous emission now follows as,

$$
\left\langle\left|M_{F I}^{(A)}\right|^{2}\right\rangle_{L / R}=\left(\frac{\hbar c k}{6 \varepsilon_{0} V}\right)\left[|\boldsymbol{\mu}|^{2} \pm 2 i c^{-1}(\boldsymbol{\mu} \cdot \mathbf{m})+c^{-2}|\mathbf{m}|^{2}\right],
$$

where the angular brackets denote a fully isotropic result. After substitution of equations (17), (18) and (21) into equation (16), the radiant intensity of emitted photons possessing left- and right- handed circular polarizations can then be derived, and recast using the formula $I=I(\Omega) / R^{2}$ to give the irradiance detected at distance $R$ :

$$
I_{L / R}=\frac{k^{4} c}{24 \pi \varepsilon_{0} R^{2}}\left[|\boldsymbol{\mu}|^{2} \pm 2 i c^{-1}(\boldsymbol{\mu} \cdot \mathbf{m})+|\mathbf{m}|^{2}\right] .
$$

As expected, the chiral differentiation evident in the mode term is observed to entail the scalar product of the electric and magnetic transition dipoles, $\boldsymbol{\mu}$ and $\mathbf{m}$. From equation (2) it emerges that the optical helicity is therefore finally expressible as;

$$
\kappa=-\frac{k^{3} V}{\pi c^{3} \varepsilon_{0}^{2}} \operatorname{Im}(\boldsymbol{\mu} \cdot \mathbf{m}) .
$$

Further implications will be considered in the later discussion.

\section{Helicity Measured in a Coupled Emitter-Detector System}

A third approach considers the system in its entirety, including a second molecular component acting as a detector of the emerging field. Since the emitted light no longer exists in the final state of this system (with it having been absorbed by the detector) the interaction between the emitter and the detector is considered to be mediated by virtual photons. Specifically, the creation and subsequent annihilation of virtual photons couples the electronic and magnetic transitions of the emitter to electronic transitions of a detector molecule. Since photodetection generally entails electric dipole excitation, it is assumed that M1 contributions to the detection process are negligible. The required matrix element for the overall process is therefore,

$$
M_{F I}^{(A-B)}=\mu_{i}^{A} \mu_{j}^{B} V_{i j}+c^{-1} m_{i}^{A} \mu_{j}^{B} U_{i j},
$$

where $(A-B)$ denotes the coupled system, in which $A$ and $B$ label the molecular emitter and detector respectively. The squared modulus of equation (24) follows,

$$
\begin{aligned}
\left|M_{F I}^{(A-B)}\right|^{2}= & \mu_{i}^{A} \mu_{j}^{B} \bar{\mu}_{k}^{A} \bar{\mu}_{l}^{B} V_{i j} \bar{V}_{k l}+c^{-2} m_{i}^{A} \mu_{j}^{B} \bar{m}_{k}^{A} \bar{\mu}_{l}^{B} U_{i j} \bar{U}_{k l} \\
& c^{-1}\left(\mu_{i}^{A} \mu_{j}^{B} \bar{m}_{k}^{A} \bar{\mu}_{l}^{B} V_{i j} \bar{U}_{k l}+m_{i}^{A} \mu_{j}^{B} \bar{\mu}_{k}^{A} \bar{\mu}_{l}^{B} U_{i j} \bar{V}_{k l}\right) .
\end{aligned}
$$

The first two terms in equation (25) will undergo no change in sign as a result of choosing the opposite enantiomer for the emitter $A$; attention therefore focuses upon the remaining terms which do change sign according to the enantiomer; the corresponding rate contributions will now be written as $\Delta \Gamma_{R / S}$. Utilizing the established forms of the two coupling tensors, the far-zone limit of the latter rate contribution is then expressible as,

$$
\begin{aligned}
\Delta \Gamma_{R / S}^{(F Z)} & =\frac{k^{4} \rho_{F}}{8 \pi c \hbar \varepsilon_{0}^{2} R^{2}}\left[\left(\mu_{x}^{A} \mu_{x}^{B}+\mu_{y}^{A} \mu_{y}^{B}\right)\left(\bar{m}_{y}^{A} \bar{\mu}_{x}^{B}-\bar{m}_{x}^{A} \bar{\mu}_{y}^{B}\right)\right. \\
& \left.+\left(\bar{\mu}_{x}^{A} \bar{\mu}_{x}^{B}+\bar{\mu}_{y}^{A} \bar{\mu}_{y}^{B}\right)\left(m_{y}^{A} \mu_{x}^{B}-m_{x}^{A} \mu_{y}^{B}\right)\right] .
\end{aligned}
$$

It is worth noting that, given electric and magnetic dipole moments that are entirely real and imaginary respectively, equation (26) reduces to zero. Physically such a result is interpreted as the inability, at least in the far-zone regime, of an achiral detector to discriminate between left- and right handed circular emission from a chiral emitter. However, in the near-zone, certainly within the span of wavelength, use of the electricdipole approximation for the detector becomes less valid. Under such conditions a displaced electric dipole can assume chiral attributes, as in the two-group (Kirkwood) model of optical activity. [28,29] Hence the following, non-zero contribution in the near-zone emerges,

$$
\begin{aligned}
\Delta \Gamma_{R / S}^{(N Z)} & =\frac{i k \rho_{F}}{4 \pi c \hbar \varepsilon_{0}^{2} R^{5}}\left[\left(\mu_{x}^{A} \mu_{x}^{B}+\mu_{y}^{A} \mu_{y}^{B}-2 \mu_{z}^{A} \mu_{z}^{B}\right)\right. \\
& \left.\times\left(m_{y}^{A} \mu_{x}^{B}-m_{x}^{A} \mu_{y}^{B}\right)\right] .
\end{aligned}
$$

It is of note that the first set of terms in brackets exhibits the form of the near-zone (or static) coupling between two electric dipoles, separated along the $z$-direction. The result (27) no longer correlates with the optical helicity of the field between the source and the detector; indeed it would otherwise contravene the principles of quantum measurement theory - since no observations are made on the system in its progression from a defined initial to a detected final state. What it does clearly show, through a lack of factorability into separate source and detector properties, is that the electronic properties of those two material components are inextricable, in the near-zone region.

\section{Conclusion}

Three distinct methods have been investigated for quantifying the helicity of circularly polarized light emitted in the electronic decay of a chiral molecule. It has been shown that results of evaluating the third Stokes parameter, and a differential rate of spontaneous emission, exhibit common features in the far-zone. Moreover, classical and QED calculations deliver results of equivalent form. The mathematical origin of chiral discrimination in the emission is a scalar product of electric and magnetic dipole moments associated with the decay transition of the emitter, and the emission has the expected dependence on the inverse square of the distance between the emitter and the point of detection.

It has been determined, however, that $S_{3}$ fails to accurately characterize chiral discrimination in the near-zone regime, as the derived expression (equation (13)) exhibits a change in sign on rotation about the optical observation axis. This failure can be considered an 
important caveat for the broad field of imaging microscopy, [30] or indeed any prospective studies aiming to extend, into the near-field regime, current far-field applications of Stokes vector analysis. [31]

Finally, an alternative, rigorous measure of optical helicity based on a coupled system incorporating both a molecular emitter and detector has been evaluated within the theoretical framework of QED. The nearfield result is noted to vary with $R^{-5}$ and exhibits features consistent with the familiar near-zone electric dipole-dipole coupling interaction, consistent with chiral dissymmetry in the coupled source-detector system.

\section{Funding Information}

The Engineering and Physical Sciences Research Council (EPSRC) (EP/K020382/1)

\section{Acknowledgements}

Helpful comments from Dr David Bradshaw are also gratefully acknowledged.

\section{References}

1. D. M. Lipkin, "Existence of a New Conservation Law in Electromagnetic Theory," J. Math. Phys. 5, 696-700 (1964).

2. S. M. Barnett, R. P. Cameron, and A. M. Yao, "Duplex symmetry and its relation to the conservation of optical helicity," Phys. Rev. 86, 013845 (2012).

3. I. Bialynicki-Birula and Z. Bialynicka-Birula, "Canonical separation of angular momentum of light into its orbital and spin parts," J. Opt. 13, 064014 (2011).

4. K. Y. Bliokh and F. Nori, "Characterizing optical chirality," Phys. Rev. 83, 021803 (2011).

5. Y. Tang and A. E. Cohen, "Optical Chirality and Its Interaction with Matter," Phys. Rev. Lett. 104, (2010).

6. G. N. Afanasiev and Y. P. Stepanovsky, "The helicity of the free electromagnetic field and its physical meaning," II Nuovo Cimento Ser. 11 109, 271-279 (1996).

7. J. L. Trueba and A. F. Ranada, "The electromagnetic helicity," Eur. J. Phys. 17,141 (1996).

8. S. M. Barnett and L. Allen, "Orbital angular momentum and nonparaxial light beams," Opt. Commun. 110, 670-678 (1994).

9. R. P. Cameron, "On the "second potential" in electrodynamics," J. Opt. 16,015708 (2014).

10. R. P. Cameron, S. M. Barnett, and A. M. Yao, "Optical helicity, optical spin and related quantities in electromagnetic theory," New J. Phys. 14, 053050 (2012).

11. M. M. Coles and D. L. Andrews, "Photonic measures of helicity: optical vortices and circularly polarized reflection," Opt. Lett. 38, 869-871 (2013).

12. M. M. Coles and D. L. Andrews, "Chirality and angular momentum in optical radiation," Phys Rev 85, 063810 (2012).

13. D. S. Bradshaw, J. M. Leeder, M. M. Coles, and D. L. Andrews, "Signatures of material and optical chirality: Origins and measures," Chem. Phys. Lett. 626, 106-110 (2015).

14. B. Ranjbar and P. Gill, "Circular Dichroism Techniques: Biomolecular and Nanostructural Analyses- A Review," Chem. Biol. Drug Des. 74, 101-120 (2009).

15. P. L. Polavarapu, "Why is it important to simultaneously use more than one chiroptical spectroscopic method for determining the structures of chiral molecules?," Chirality 20, 664-672 (2008).

16. H. G. Brittain, "Applications of chiroptical spectroscopy for the characterization of pharmaceutical compounds," J. Pharm. Biomed. Anal. 17, 933-940 (1998).

17. R. Carr, N. H. Evans, and D. Parker, "Lanthanide complexes as chiral probes exploiting circularly polarized luminescence," Chem. Soc. Rev. 41, 7673 (2012)

18. J. P. Riehl and F. S. Richardson, "Circularly polarized luminescence," Methods Enzymol. 226, 539-552 (1993).
19. J. P. Riehl and F. S. Richardson, "Circularly polarized luminescence spectroscopy," Chem. Rev. 86, 1-16 (1986).

20. A. Zangwill, Modern Electrodynamics (Cambridge University Press, 2013).

21. D. H. Goldstein, Polarized Light, 3rd ed. (CRC Press, 2011).

22. P. D. Drummond and M. Hillery, The Quantum Theory of Nonlinear Optics (Cambridge University Press, 2014).

23. J. Jackson, Classical Electrodynamics, 3rd ed. (Wiley, 1998).

24. G. J. Daniels, R. D. Jenkins, D. S. Bradshaw, and D. L. Andrews, "Resonance energy transfer: The unified theory revisited," J. Chem. Phys. 119, 2264-2274 (2003).

25. G. Grynberg, A. Aspect, and C. Fabre, Introduction to Quantum Optics (Cambridge University Press, 2010).

26. D. L. Andrews and T. Thirunamachandran, "On three-dimensional rotational averages," J. Chem. Phys. 67, 5026-5033 (1977).

27. G. Wagnière, "The Evaluation of Three-dimensional Rotational Averages," J. Chem. Phys. 76, 473-480 (1982).

28. J. G. Kirkwood, "On the Theory of Optical Rotatory Power," J. Chem. Phys. 5, 479 (1937).

29. L. Barron, Molecular Light Scattering and Optical Activity, 2nd ed. (Cambridge University Press, 2009).

30. D. A. Schmidt, I. Kopf, and E. Bründermann, "A matter of scale: From far-field microscopy to near-field nanoscopy," Laser Photonics Rev. 6, 296-332 (2012).

31. N. Mazumder, C.-W. Hu, J. Qiu, M. R. Foreman, C. M. Romero, P. Török, and F.-J. Kao, "Revealing molecular structure and orientation with Stokes vector resolved second harmonic generation microscopy," Methods 66, 237-245 (2014). 